\title{
PCV Measurement for Biomass Assessment in Suspension Culture of Insect Cells
}

\author{
Dong-Chen LIU ${ }^{1}$, Qiu-Ling XIE ${ }^{2, a, ~ * ~}$ \\ ${ }^{1}$ College of Life Science and Technology, Jinan University, Guangzhou 510632, China \\ Guangdong Provincial Key Laboratory of Bioengineering Medicine, Guangzhou \\ 510632, China \\ ${ }^{2}$ National Engineering Research Center of Genetic Medicine, Guangzhou \\ 510632, China \\ aemail:x_qiuling@hotmail.com \\ ${ }^{*}$ Corresponding author
}

Keywords: PCV measurement, Biomass assessment, Insect cell culture.

\begin{abstract}
In this study, we present a new approach for biomass assessment in insect cell culture using a disposable microcentrifuge tube, which is composed with an upper chamber for sample loading and a lower $5 \mu \mathrm{L}$ capillary for cell collection during centrifugation. The height of the cell pellet within the capillary is measured and expressed as the packed cell volume (PCV). PCV method showed an excellent correlation with cell counting measurement, but with lower average of the errors $(3.3 \%)$ compared to manual cell counting (12.4\%). PCV method was shown to be statistically reliable and more precise, rapid. And it also demonstrates the potential use of this method to monitor the changes in insect cell culture that can affect cell size, for example as seen under baculovirus infection.
\end{abstract}

\section{Introduction}

Biomass is a critical parameter for the evaluation of bioprocess of insect culture like in microbial system and mammalian cells. The most popular approach for biomass assessment in suspension culture of insect cells is cell counting using dye exclusion, which has limited accuracy and is time-consuming and tedious. There are some automated particle counters applied for cell counting now. These instruments have been utilized in industrial bioprocess. But they are too expensive for most academic labs and the repeated research in labs. Moreover, cell aggregates can induce major biomass determination errors when using both methods above mentioned. Packed cell volume (PCV) measurement of the cells may be a better approach for biomass assessment.

PCV or Packed Cell Volume describes the volume that is occupied by a cell pellet after centrifugation. PCV or hematocrit is widely employed for blood analysis [1]. And it is a useful parameter for plant cell perfusion culture and design of perfusion bioreactor for plant cell culture [2,3]. A new disposable microcentrifuge tube is used to measure the biomass of mammalian cells culture such as CHO, HEK and NSO cells because of the low cell density in mammalian cell culture [4]. This tube is composed with two chambers, and the upper chamber has an external diameter which is identical to a standard $1.5 \mathrm{~mL}$ microcentrifuge tube so that it can load up to $1 \mathrm{~mL}$ of cell suspension, while the lower one is a capillary with a volume of $5 \mu \mathrm{L}$ (Fig. 1).

In this study, we tried to assess the biomass of insect cell culture using PCV measurement with the new disposable microcentrifuge tube to set up a faster and 
more precise method for suspension insect cell culture.

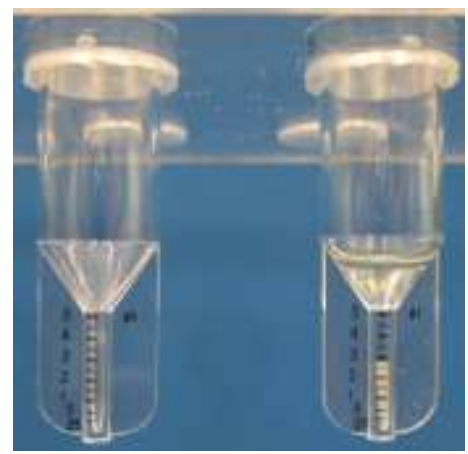

Figure 1. The mini PCV tubes. The well-mixed suspension culture can be loaded into the upper part of the tube. The cell pellet would be analysis after centrifugalization

\section{Materials and Methods}

\section{Cell Culture}

Insect cell Sf9 was grown in medium SF900II (Gibco) in $50 \mathrm{~mL}$ single-use TubeSpin bioreactors (Techno Plastics Products AG, NEST, China) [5]. The cells were incubated in a shaker at $28^{\circ} \mathrm{C}$ with orbital agitation of $200 \mathrm{rpm}$. The cells were passaged every 4-5 days at densities of $5 \times 10^{5}$ cells $/ \mathrm{mL}$.

\section{Cell Counting and PCV Determination}

Cell density and viability were assessed with a hemocytometer using the Trypan blue exclusion method. The cell density included both viable and non-viable cells and was therefore referred to as total cell density.

For PCV measurements, $100 \mu \mathrm{L}$ of a well-mixed suspension culture were transferred directly into a mini PCV tube manufactured by Techno Plastics Products AG, Trasadingen, Switzerland. The tubes were centrifuged in a microcentrifuge (Model 5417C, Eppendorf AG, Hamburg, Germany) for $1 \mathrm{~min}$ at 2,500 g (5,000 rpm). The total volume of the cell pellet was either read directly by naked eye, using the volume indications on the tube as guide or with a TPP Easy Read Device manufactured by TPP-US Company. The Easy Read device is a blue metal ruler with a gray metal guide includes both a magnifying glass as well as the tube holder. The bottom of the PCV tube is placed into the holder and rests against the slant of the device. The tube then slides up and down that slant as you move the gray metal piece across the ruler. Once the top of your pellet is flush with the ruler, you can read the measurement through the magnifying glass [6].

\section{Results and Discussion}

The cells were cultivated at $28^{\circ} \mathrm{C}$ and triplicate samples was taken every day for manually cell counting and PCV measurement. The growth curve was got on base of the cell density data and PCV data. The experiment was repeated three times and the similar results were gotten. The growth curves according to the cell density data had the same trend with those based on PCV measurement (Fig. 2), meaning PCV assessment could reflect the biomass and growth kinetics of insect cell cultures. 


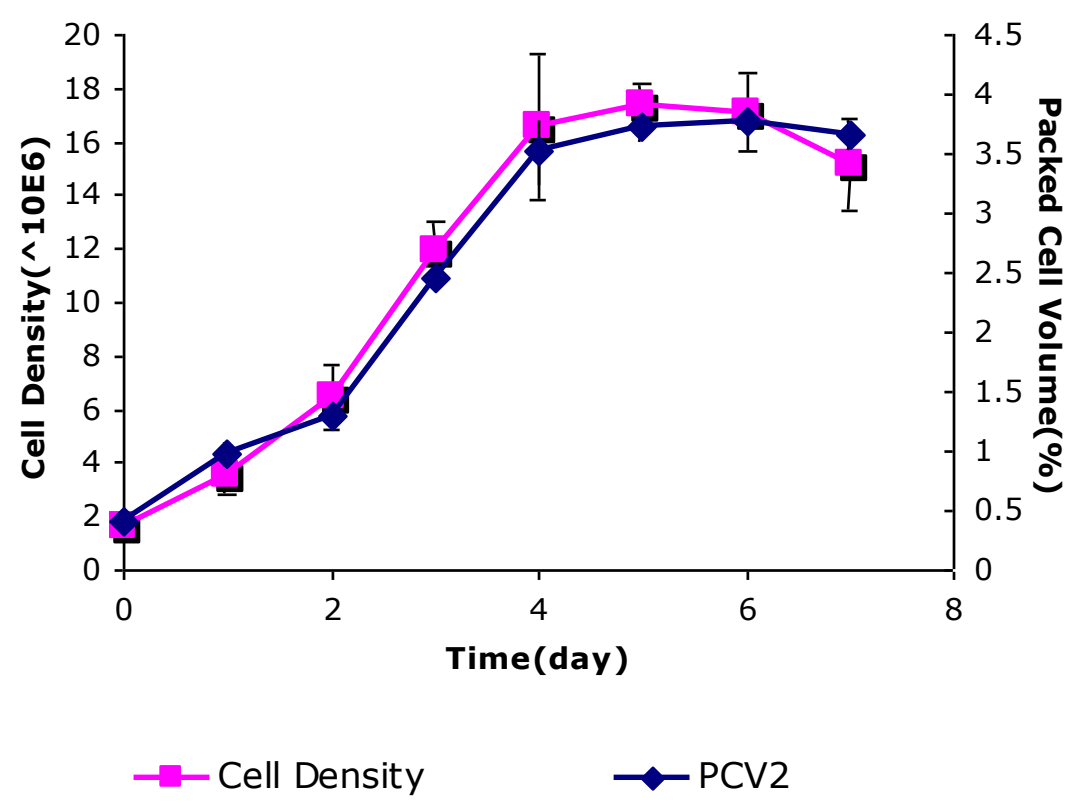

Figure 2. Growth curves of insect cell culture with cell density and PCV assessment

The PCV assessment offers several advantages over counting cells manually with a hemacytometer. There is no need to dilute and stain the samples and multiple samples can be counted very quickly. More important, it can provide more accurate and cleaner data. The variation of cell densities was much bigger than that of PCVs. The average CV\% of cell counting data is about $12.4 \%$ while that of PCV measurement is only $3.3 \%$ (Fig. 3).

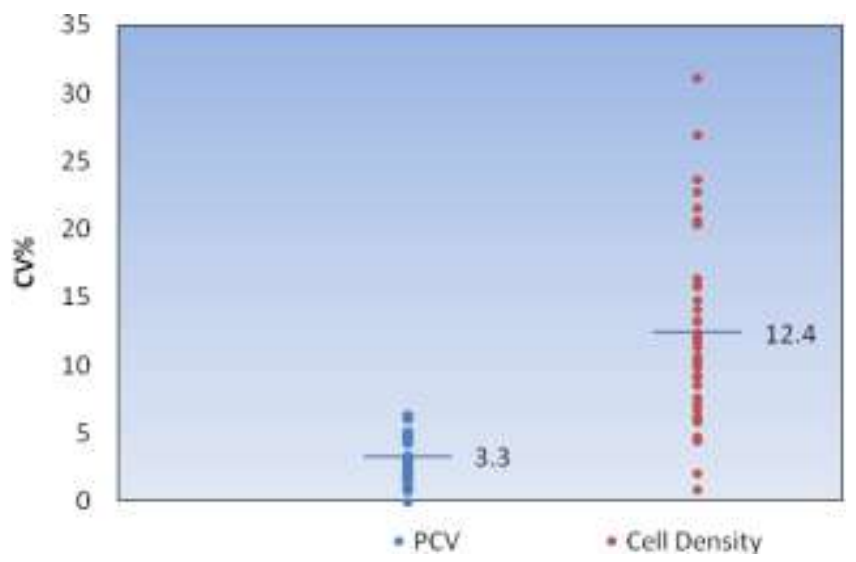

Figure 3. Comparing the CV\% of cell counting with PCV measurement of the same sample

It is may be generally useful to be able to convert PCV data into a cell density even if biomass data as expressed in a percentage of the total volume of the bioreactor representing the volume of cells obtained after a centrifugation step is sufficient as an independent parameter. Such a conversion can be done by determining the packed cell volume of a given number of cells and thus obtaining the average volume per cell, including the small volume of intercellular liquid - when packed under the condition of the centrifugation utilized. We judge that the volume of the liquid between cells is minimal. We also assume that on average the volume of cells is constant.

It is reported that the mean diameter of insect cell Sf9 is about $18.3 \mu \mathrm{m}$ [6]. Thus the volume of 1 million cells is about $3.2 \mu \mathrm{L}$. Corresponding to a PCV of $0.32 \%$ if one million cells had been loaded in a volume of $1 \mathrm{ml}$ of medium into the tube, it is 
known that there are size differences among the cells and the size of insect cell Sf9 sometimes ranges from $12 \mu \mathrm{m}$ to $28 \mu \mathrm{m}$, most of them from 16 to $20 \mu \mathrm{m}$. Thus we chose the second approach and got the relationship between the cell density and PCV as Fig. 4. The linear regression gives the correlation between the two biomass assessment methods as 1million cells=0.28 PCV. In Fig. 5 the plotted data are given and the regression graph provided now a \% PCV value of 0.42 for a cell density of 1 million cells $/ \mathrm{mL}$.

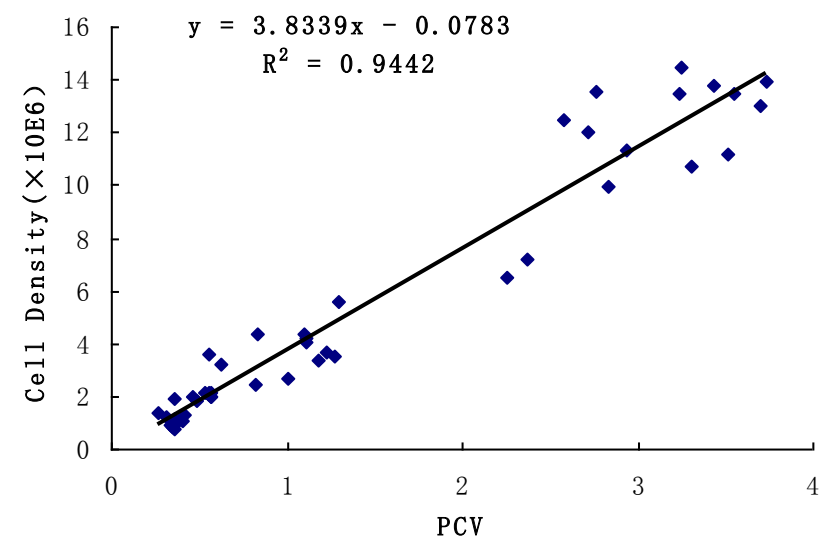

Figure 4. Correlation between the PCV and cell density of new defrost cell

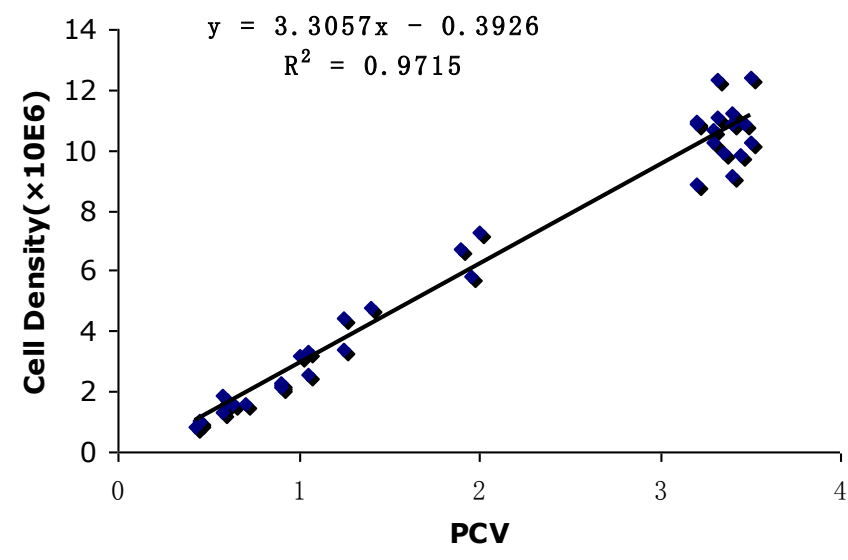

Figure 5. Correlation between the PCV and cell density after cell passages for 4months

It is also found that the correlation between cell density and PCV would change after long time passages. After 4 months of passages, the relationship of insect cell density and PCV is as Fig. 5 shows that 1 million cells $/ \mathrm{mL}$ is calculated to be 0.4213 $\mathrm{PCV}$ according to the regression equation, meaning the cells became bigger. It is testified under microscope that the cells became bigger after long time of passages (Fig. 6).

The size of cells will be changed because of the cell age, change of culture condition and other pathological reason such as the infection of virus. For insect cell $\mathrm{Sf} 9$, which is always used as the host for infection of Baculovirus to express the recombinant protein, the size of cells before and after infection is different. The change in average diameter of insect cell Sf9 during process of infection could be used to determine the baculovirus titer and the time of maximum protein production and, thus, optimal harvest time [7,8]. So the size of cell is a critical parameter for the culture of insect cell process. Usually, the cell size should be measured under microscope or with special instrument, which is tedious and time-cost. Now, it can be monitored by calculating the correlation of cell density and PCV. 


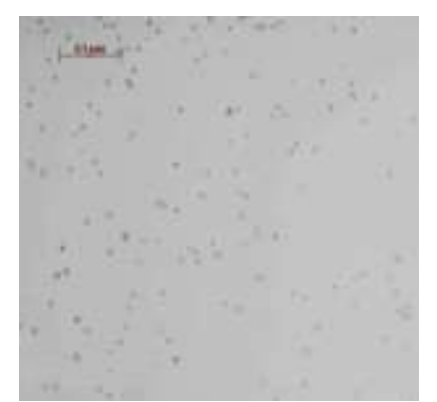

\section{A}

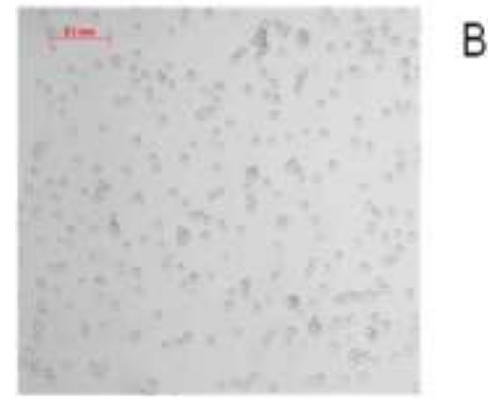

Figure 6. Insect cell Sf9 images. (A) the cells newly defrosted;(B) the cells after passaging for 4months.

\section{Conclusions}

The here presented PCV method allows statistically reliable measurements of the total biomass including cell fragments and aggregates and offers more accurate, more rapid approach for biomass assessments of insect cell and other cell lines. Moreover, the study also demonstrates the potential use of this method to monitor the changes in insect cell culture that can affect cell size, for example as seen under baculovirus infection. The method does not allow the assessment of viability, a drawback which is acknowledged. However efforts are underway to develop a "reader" for the PVC tubes which through image analysis and staining of cells would allow obtaining insights into viability parameters. Overall, the higher precision and the ease of use of the PCV tubes appears to us a valuable additional tool for a rapid assessment of the biomass of insect cell cultures.

\section{Acknowledgement}

We thank Prof. Florian Wurm and Dr. Yashas Rajendra of EPFL, Switzerland for technical help. This study was financially supported by National Major Scientific and Technological Special Project for "Significant New Drugs Development" (2012ZX09202-301-001) and Major Scientific and Technological Special Project of Guangdong Province (2012A080800008, 2012A080202014).

\section{Reference}

[1] BRIAN S. BULL, KAREN L. HAY. Is the Packed Cell Volume (PCV) Reliable? Laboratory Hematology 2001, 7:191-196

[2] WE1 WEN SU, AND RENEE ARIAS .Continuous Plant Cell Perfusion Culture: Bioreactor Characterization and Secreted Enzyme Production. JOURNAL OF BIOSCIENCE AND BIOENFINMRING2003,95(1): 13-20.

[3] C. De Dobbeleer, M. Cloutier, M. Fouilland, R. Legros, M. Jolicoeur. A High-Rate Perfusion Bioreactor for Plant Cells. Biotechnology and Bioengineering, 2006 Vol. 95( 6): 1126-1137

[4] Matthieu Stettler, Nicolas Jaccard, David Hacker, Maria De Jesus, Florian M. Wurm, Martin Jordan. New Disposable Tubes for Rapid and Precise Biomass Assessment for Suspension Cultures of Mammalian Cells. Biotechnology and Bioengineering 2006,95(6):1228-1233

[5] De Jesus M, Girard P, Bourgeois M, Baumgartner G, Jacko B, Amstutz H, Wurm 
FM. 2004. TubeSpin satellites: A fast track approach for process development with animal cells using shaking technology. Biochem EngJ 17(3):217-223.

[6]http://www.tpp-us.com/Blogs/BlogB/index.cfm/2009/8/28/Using-the-TPP-Easy-Re ad-Device

[7]http://www.nexcelom.com/Literature/Appl\%20Note\%2007007\%20Insect\%20Cells .pdf

[8] Janakiraman V, Forrest WF, Seshagiri S. Estimation of baculovirus titer based on viable cell size.Nat Protoc. 2006;1(5):2271-6.

[9] Laura Sander \& Anna Harrysson. Using cell size kinetics to determine optimal harvest time for Spodoptera frugiperda and Trichoplusia ni BTI-TN-5B1-4 cells infected with a baculovirus expression vector system expressing enhanced green fluorescent protein Cytotechnology (2007) 54:35-48 